\title{
Blazhko Effect and Magnetic Field in RR Lyrae
}

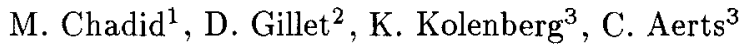

\begin{abstract}
The recent detection of a frequency splitting around the pulsation frequency and its harmonics points towards the magnetic model to explain the Blazhko effect. Here we show that it is urgent to confirm with modern observational techniques the existence of the magnetic field in RR Lyrae.
\end{abstract}

\section{Introduction}

Single periodic RR Lyrae stars repeat their light and velocity curves from cycle to cycle with amazing regularity. Nevertheless, about one third of the observed RR Lyrae stars present a long-term modulation of their pulsation which is called Blazhko effect. More exactly, this consists in quasi-periodic changes of the amplitude and shape of the luminosity and radial velocity curves while the pulsation period remains constant. The physical origin of this effect is not yet completely understood.

The existence of an observable magnetic field in RR Lyrae has been detected several times. First Babcock (1958) and more recently Romanov, Udovichenko, \& Frolov $(1987,1994)$ put into evidence that the magnetic field of RR Lyrae varies with a period equal to the pulsation one and an amplitude around $1.5 \mathrm{kG}$, and that the mean magnetic field strength presents a periodic variation with the phase of the Blazhko effect. These results seriously point out that the Blazhko effect may be associated with the magnetic activity of the star. Nevertheless, up to now, these magnetic observations, which were based on photographic plates, have never been confirmed with modern observational techniques.

\section{A Connection with the Blazhko Effect?}

As already discussed by Kovács (1995), three plausible models are presently proposed as an explanation for the Blazhko effect. One of them is the oblique magnetic pulsator model (Cousens 1983; Shibahashi \& Takata 1995; Shibahashi 2000). All versions of the oblique pulsator model in use so far are developed in the linear pulsation approach. The effects of the magnetic field and of the rotation are treated as small perturbations, and the effect of the centrifugal force is

\footnotetext{
${ }^{1}$ European Southern Observatory, Alonso de Cordova, 3107, P.O. 19001, Santiago, Chile

${ }^{2}$ Observatoire de Haute-Provence, Saint-Michel-l'Observatoire, F-04870, France

${ }^{3}$ Instituut voor Sterrenkunde, Katholieke Universiteit Leuven, Belgium
} 
neglected. This results both in an eigenfrequency shift and in the deformation of the eigenfunction of the radial fundamental mode. The interpretation of the Blazhko effect is as follows: the radial eigenfunction excited by the $\kappa$-mechanism is deformed, mainly by the Lorentz force, to have additional nonradial components whose symmetry axis coincides with the magnetic axis. The latter is assumed to be inclined ("oblique") to the rotation axis of the star. As the star rotates, the aspect angle of the nonradial components varies and then these components manifest themselves as the long-term modulation of the luminosity variation of $R R$ Lyrae stars. Essential in this model is the dependence of the Blazhko amplitude upon the magnetic field strength. A magnetic field of order $1 \mathrm{kG}$ is needed for this model.

The oblique magnetic pulsator model for a nonlinear radial pulsation mode is compatible with the periodic variations of the magnetic field over the pulsation period and its average intensity over the Blazhko cycle, as well as the appearance of the frequency splitting around the harmonics of the main frequency. This model, however, predicts a quintuplet frequency structure, while at present recent observations only allow the unambiguous detection of a triplet (Chadid et al. 1999). The two other models (Kovács 1995) do not require the existence of a magnetic field and as such cannot be confronted with the findings of Romanov et al. $(1987,1994)$.

\section{Conclusion}

To know if the oblique magnetic pulsator model is plausible to explain the Blazhko effect, it is urgent to re-measure the magnetic field of RR Lyrae with a modern spectropolarimeter such as MUSICOS (Donati et al. 1999) which presently can detect a magnetic field strength around $50 \mathrm{G}$ for the disk-averaged line-of-sight component.

\section{References}

Babcock, H. W. 1958, ApJS, 3, 141

Chadid, M., Kolenberg, K., Aerts, C., \& Gillet, D. 1999, A\&A, in press

Cousens, A. 1983, MNRAS, 203, 1171

Donati, J.-F., Catala, C., Wade, G. A., et al. 1999, A\&AS, 134, 149

Kovács, G. 1995, A\&A, 295, 693

Romanov, Yu. S., Udovichenko, S. N., \& Frolov, M. S. 1987, Sov. Astr. Lett., 13,29

Romanov, Yu. S., Udovichenko, S. N., \& Frolov, M. S. 1994, Bull. Spec. Astrophys. Obs., 38,169

Shibahashi, H. 2000, in these proceedings, p. 299

Shibahashi, H. \& Takata, M. 1995, in ASP Conf. Ser. Vol. 83, Astrophysical Applications of Stellar Pulsation, ed. R. S. Stobie \& P. A. Whitelock (San Francisco: ASP), 42 